\title{
A large fiber sensor network for an acoustic neutrino telescope
}

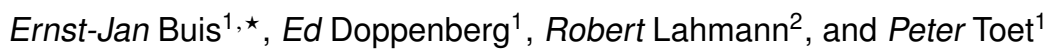 \\ ${ }^{1}$ TNO Technical Sciences, P.O. Box 155, 2600 AD Delft, The Netherlands \\ ${ }^{2}$ Erlangen Centre for Astroparticle Physics, University of Erlangen, Erwin-Rommel-Str. 1, 91058, Erlangen, \\ Germany
}

\begin{abstract}
The scientific prospects of detecting neutrinos with an energy close or even higher than the GKZ cut-off energy has been discussed extensively in literature. It is clear that due to their expected low flux, the detection of these ultra-high energy neutrinos $\left(E_{v}>10^{18} \mathrm{eV}\right)$ requires a telescope larger than $100 \mathrm{~km}^{3}$. Acoustic detection may provide a way to observe these ultra-high energy cosmic neutrinos, as sound that they induce in the deep sea when neutrinos lose their energy travels undisturbed for many kilometers. To realize a large scale acoustic neutrino telescope, dedicated technology must be developed that allows for a deep sea sensor network. Fiber optic hydrophone technology provides a promising means to establish a large scale sensor network [1] with the proper sensitivity to detect the small signals from the neutrino interactions.
\end{abstract}

\section{Introduction}

The measurements of neutrinos with energies higher than what has been detected so far may provide input in one of the long standing issues in astrophysics, which is the mystery of cosmic rays and the mechanism of their acceleration to the highest energies measured [2]. To detect the neutrinos that originate from the GKZ cut-off, a neutrino telescope is required with a detection volume of at least $100 \mathrm{~km}^{3}$. To establish such as large detection volume one could employ the technique of acoustic detection of neutrinos [3-5]. In this paper we motivate the use of optical sensor networks as a building block of an acoustic neutrino telescope and provide an overview of the research and development of the fiber hydrophone technology at TNO.

\section{Acoustics neutrino telescope concept}

As the absorption length of sound in seawater is as large as $\sim 1 \mathrm{~km}$ (at an acoustic frequency of $25 \mathrm{kHz}$ ), the acoustic detection of high energy cosmic rays provides a means to create a truly large neutrino telescope. An example of an acoustic signal is shown Fig.11(a), where a typical bipolar pulse is plotted that has an amplitude at the order of a $\mathrm{mPa}$. The dominant frequency components of this signal are found between 1 and $40 \mathrm{kHz}$.

To discriminate the acoustic signal from background noise, a telescope is required that is equipped with many (>1000) hydrophones. It means that a cost-effective technology needs to developed that

^e-mail: ernst-jan.buis@tno.nl 
can be deployed on industrial scale, but that provides the proper sensitivity to detect the mPa signals at the same time. Optical fiber hydrophone technology might provide a solution here as the hydrophones can produced and deployed on a cost effective way. Moreover, the technology is based on (passive) optical fibers, so that (in contrast to conventional piezo-hydrophones) no electronics (or electrical power) is needed at the sensitive parts of the system. Finally, since the signals from the hydrophones may travel through optical fibers unattenuated for many kilometers, the technology is suited for a large sensor network. A concept of an acoustic neutrino telescope is depicted in Fig. 1 (b), where a 3D grid of hydrophone strings is deployed a depth of several km, similar to the concept of KM3NeT.

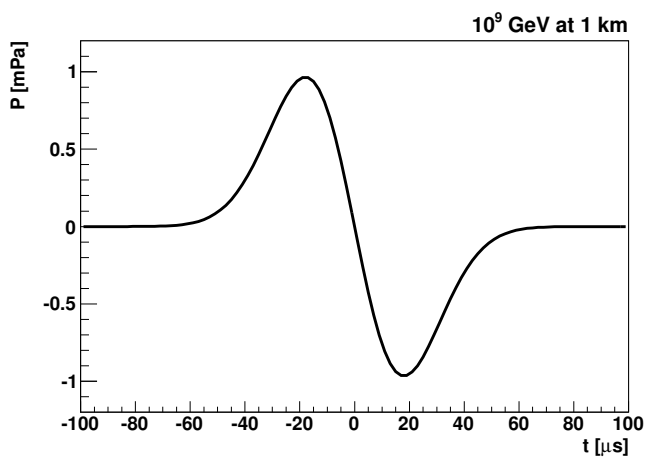

(a)

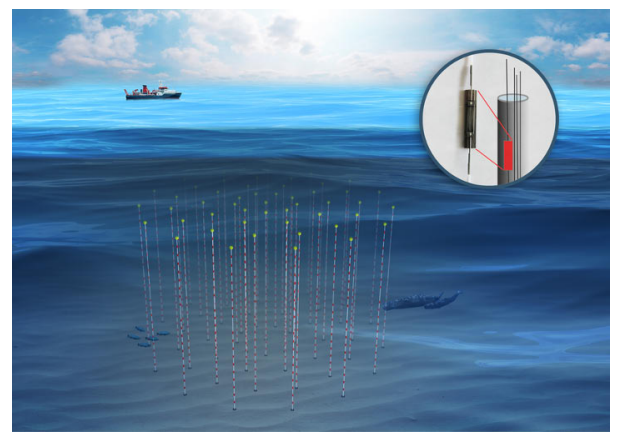

(b)

Figure 1. (a) Typical acoustic signal from a energetic neutrino induced particle shower. Adapted from [6]. In (b) an underwater neutrino telescope is schematically depicted.

\section{Optical fiber hydrophone technology}

The optical fiber hydrophone that has been developed at TNO is explained in detail in [7, 8] and shown in Fig. 2. A hydrophone consists of a mechanical transducer that converts a pressure wave in to strain in the optical fiber. At the position of the transducer a fiber laser structure is locally inserted in the fiber, which schematically depicted in figure Fig.2(b). A fiber laser consists of two fiber Bragg gratings (FBG) and a gain medium that includes a high concentration of Erbium ions. The cavity that is defined by the two FBGs at either side is pumped using an external pump laser that can be located at a large distance. The emitted laser light is highly coherent as it is defined by the cavity and the reflectivity of the FBGs In Fig. 2 (b) the reflectivity spectrum of the FBGs is shown. The strain that is induced on the cavity by the transducer will result in a wavelength shift of the emitted laser light. Finally, this shift is a measure for the acoustic signal.

\section{Interrogation}

Using the optical hydrophones as discussed above a sensor network can be created by implementing many transducers on a single fiber as shown in Fig. 3. On a single fiber about 10 hydrophones can be housed [8]. A dedicated interrogator has been developed that is able to detect minute wavelength shifts. The interrogator is based on a $3 \times 3$ interferometer, which is used to determine the wavelength shift of the light that is coupled into the interrogator. The output of the $3 \times 3$ coupler consists of three 


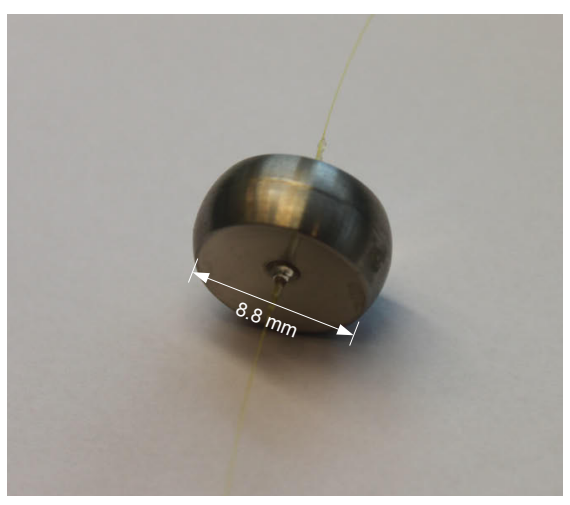

(a)
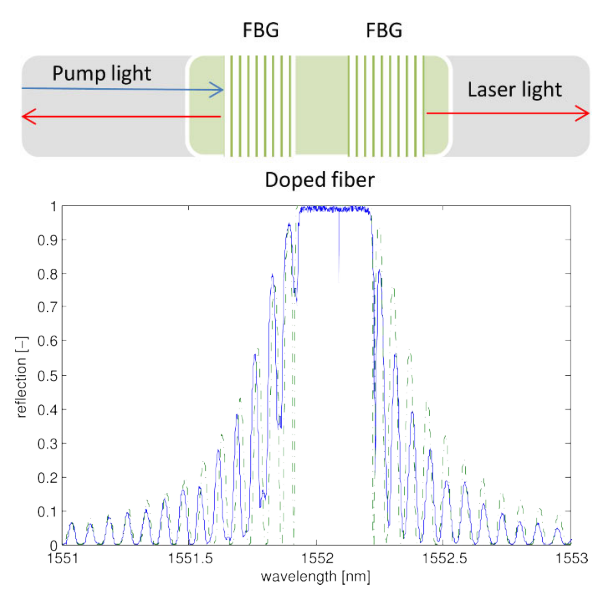

(b)

Figure 2. Optical hydrophone technology: in (a) a transducer is shown attached to a optical fiber. The transducer is designed to have a strain sensitivity of $\eta=70 n \varepsilon / m P a$. In (b) the fiber laser cavity is shown that is located in the optical fiber at the position of the transducer. Also shown is the reflectivity spectrum of the FBGs.

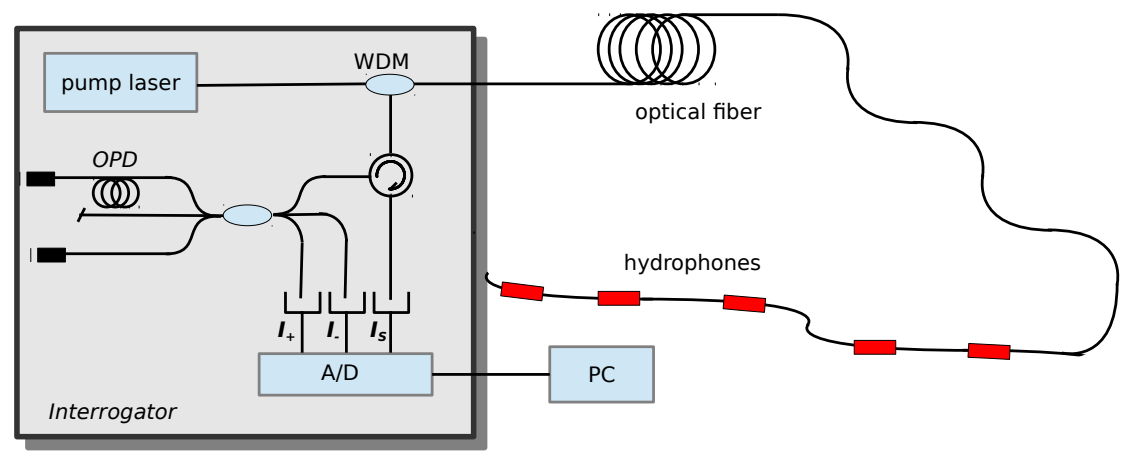

Figure 3. Concept of the optical fiber sensor network: An optical fiber with several fiber lasers and sensors are read out using an interrogator. Once deployed in the deep-sea, several of these strings make up a neutrino telescopes.

light intensities $\left(I_{x}\right)$ as indicated in Fig. 3 Note that the outputs of the three arms are identical, but each is shifted in phase over $\frac{2}{3} \pi$ as given in the following expression.

$$
\begin{aligned}
I_{S}(O P D) & =I_{0}\left(1+V \cos \left(\frac{2 \pi}{\lambda} O P D\right)\right) \\
I_{+}(O P D) & =I_{0}\left(1+V \cos \left(\frac{2 \pi}{\lambda} O P D+\frac{2 \pi}{3}\right)\right) \\
I_{-}(O P D) & =I_{0}\left(1+V \cos \left(\frac{2 \pi}{\lambda} O P D-\frac{2 \pi}{3}\right)\right)
\end{aligned}
$$

where $I_{0}$ is the normalized amplitude of the signal and $V$ is the visibility. Using the phase shift and the intensity of each arm, the optical path length $(O P D)$ is given by the following equation:

$$
O P D=\frac{\lambda}{2 \pi} \arctan \left(\sqrt{3} \frac{I_{+}-I_{-}}{2 I_{s}-I_{+}-I_{-}}\right)
$$


Because the optical path difference $(O P D)$ is known, the wavelength shift of each hydrophone in the string can be accurately determined when the three output intensities are measured. In Fig. 4 the performance of the interrogator is plotted as a function of the (acoustic) frequency. One can see from the plot that the strain noise is at the order of $10^{-13} \varepsilon / \sqrt{H z}$, while the strain sensitivity of the transducer is around $\eta=70 n \varepsilon / \mathrm{mPa}$. As an example, a measurement of a synthetic pulse (after filtering) is shown Fig. 4(b), in which the typical bipolar structure is clearly visible.

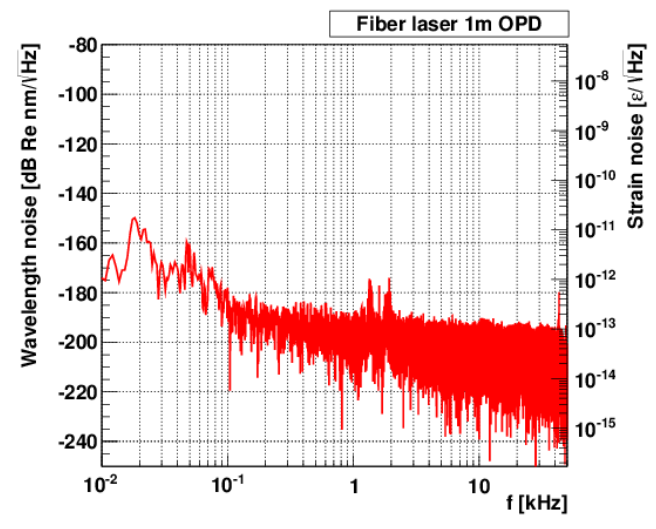

(a)

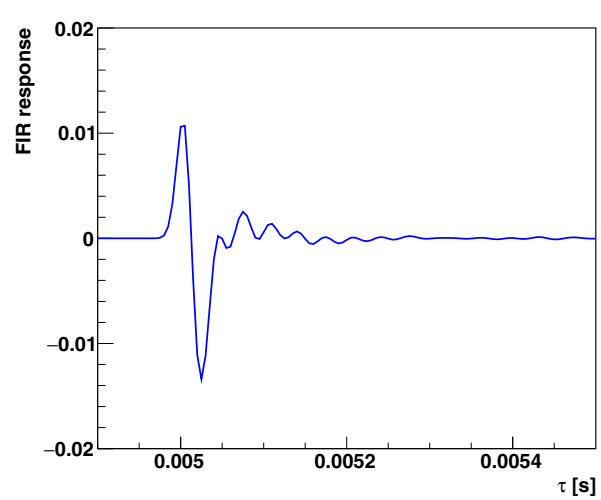

(b)

Figure 4. (a) The noise performance of the interrogator. In (b) an example of a measured pulse is shown after filtering. While a large part of the mechanical resonance was filtered, the graph still shows some ringing.

\section{Conclusions}

This conference has shown that the perspectives for both radio and acoustic detection of high energetic neutrino are bright as new technologies to detect these particles become available. It is of high importance that both detection methods will be pursued for many reasons, such as cross calibrations and background removal. The technology for the acoustic detection of neutrinos as discussed in this paper has the potential to be implemented on large scale with the proper sensitivity and is hence suitable for a large volume neutrino telescope. Next steps in the development are targeted on the multiplexing of the output of a large number of hydrophones.

\section{References}

[1] E.J. Buis et al., Journal of Instrumentation 9, C03051 (2014)

[2] E. Waxman, Neutrino Astronomy: What did we learn, Where should we aim?, (2016), these proceedings

[3] G.A. Askaryan, Zemlia i Vselennaia 1, 13 (1979)

[4] J.G. Learned, Phys. Rev. D 19, 3293 (1979)

[5] R. Nahnhauer, Nucl. Instr. Meth. A 692, 58 (2012)

[6] R. Lahmann, Ph.D. thesis, Friedrich-Alexander Universität Erlangen (2011)

[7] E.J. Buis et al., Design studies for a neutrino telescope based on optical fiber hydrophones, (2015), in proceedings of the " $34^{\text {th }}$ International Cosmic Ray Conference"

[8] V.M. Baas et al., Fiber laser design and measurements for fiber optical hydrophones, (2015), in proceedings of the " $34^{\text {th }}$ International Cosmic Ray Conference" 